$\begin{array}{ll}\text { Research Square } & \begin{array}{l}\text { Preprints are preliminary reports that have not undergone peer review. } \\ \text { They should not be considered conclusive, used to inform clinical practice, } \\ \text { or referenced by the media as validated information. }\end{array}\end{array}$

\title{
Dental caries experience in adults: a cross-sectional community survey within Ethiopia
}

Birke Bogale ( $\sim$ birke_bogale.lema@kcl.ac.uk)

St Paul's Hospital Millennium Medical College, King's College London https://orcid.org/0000-0001-6725-8573

Fasikawit Engida

Addis Ababa University

Charlotte Hanlon

Addis Ababa University

Martin J Prince

King's College London

Jennifer E Gallagher

King's College London

Research

Keywords: Dental caries, Dental caries experience, Oral health, Health System, Ethiopia

Posted Date: May 7th, 2020

DOI: https://doi.org/10.21203/rs.3.rs-26418/v1

License: @) (7) This work is licensed under a Creative Commons Attribution 4.0 International License. Read Full License 


\section{Abstract}

Background Ethiopia is a large and diverse developing sub-Saharan African country with increasing prevalence of non-communicable diseases, including oral conditions. Oral health and dental care have been given little consideration, and there is limited information relating to population oral health and use of dental services in the country. The aim of this study was to examine the burden and impact of dental caries experience and investigate access to dental care amongst adults within Ethiopia.

Methodology This community-based oral health survey is a baseline study for the ASSET - Health System Strengthening in sub-Saharan Africa project undertaken in the Butajira area, south-central Ethiopia. A stratified random sample of households and individuals were participated in the study. The survey was informed by the World Health Organization (WHO) Oral Health Survey Methods (5th ed.) at International Caries Detection and Assessment System (ICDAS) threshold 4 and the UK Adult Dental Health Survey 2009. Face-to-face interviews and clinical dental examinations were conducted. The data were analysed using descriptive statistics; and a binary logistic regression model was built to assess the association of dental caries and predictor variables: demographics, health behaviours, quality of life, dentition status and consequences of untreated caries data in adults ( $\geq 18$ years).

Result Most of the study population ( $n=626)$ were female (63.9\%), married $(71.4 \%)$ and Muslim (76.0\%). Just over half (53.2\%) lived in rural areas and many (44.4\%) had no formal education. A majority (74.0\%) reported never attending a dental service. Sixty percent $(\mathrm{n}=377)$ of the adults had experienced dental caries, $88.0 \%(n=332)$ of whom had untreated carious teeth. In the fully adjusted logistic regression model; increasing age, dental attendance and Khat chewing had a positive significant association with dental caries experience, whilst education status was negatively associated $(p<0.05)$.

Conclusion This study demonstrated a high burden of dental caries and considerable impact resulting from untreated disease in this population of adults. There was also evidence of social inequity and limited overall access to dental care, highlighting the need for health system strengthening.

\section{Background}

Oral health is an integral element of good health and wellbeing as it facilitates essential functions, most notably: eating, speaking, smiling, and socialising (1). Oral conditions, especially dental caries and periodontal diseases are considered major global public health problems (2-4). Although mostly preventable and treatable, they can be life threatening. About $90 \%$ of the world population face at least one form of oral condition sometime in their lives and oral conditions have been associated with adverse socioeconomic impact in both adults and children irrespective of their gender (5). Overall, untreated caries in secondary teeth, primary teeth and severe chronic periodontitis are the top three most prevalent oral disorders (6).

Oral health in Africa has been considered a low priority in relation to communicable diseases. However, non-communicable diseases (NCDs), under which common oral conditions are classified, are increasing across the continent (7). In a 2015 report on the severity and prevalence of oral conditions in the Africa/Middle East Region (AMER), reliable data were found to be scarce, but there was evidence of an increasing burden of oral diseases with related morbidity. There are substantial oral health inequalities in both high- and low-income nations and the prevalence of dental caries, periodontal diseases, orofacial trauma and oral cancer, as well as Noma, are increasing (8). This is mainly related to specific risk factors associated with nutritional transitions transition towards the 'Western diets' involving increased sugar consumption - together with low fluoride availability and inadequate dental care, in addition to other general factors including age, socioeconomic status and area of residence (9).

Ethiopia is a large, diverse low-income sub-Saharan African country in the east African region with rich natural resources and history. It has one of the world's fastest growing economies with a rapid real domestic product growth despite the low Gross Domestic Product (GDP) per capita (10). Agriculture engages nearly $85 \%$ of the country's workforce and many economic activities depend on it, with most of the population living in rural areas. Considerable economic and health inequalities exist between the urban and rural populations nationally (11). Ethiopian oral health data are notably scarce as there are no communitybased epidemiological oral health surveys. However, local hospital and school-based cross-sectional studies suggest untreated dental caries present a problem. First, in two local studies in north-western Ethiopia, untreated dental caries were identified in about one-third and one-fifth of schoolchildren aged 714 and 6-15 years, respectively, whereby the disease was positively associated with toothache, and negatively associated with oral hygiene, household income and paternal education $(12,13)$. Second, findings from hospital attendees, involving children and adults, indicate that dental caries, which affected three-quarters of attendees, was the most frequent reason for presentation at the dental service $(14,15)$.

Healthcare in Ethiopia is improving, and the government has made substantial investment aligned with the sustainable development goals to strengthen the public healthcare services to improve population health (16); however, change will take time. Access to health services in general is harder for the rural population $(17,18)$. Furthermore, dental services in particular are very limited, predominantly found in the private sector as well as in secondary and tertiary referral public hospitals which are largely located in the cities and bigger towns $(19,20)$, with implications for patient access.

Africa itself possesses only $1 \%$ of the global dental workforce $(21,22)$ and has a dentist: population with a ratio of around $1: 40,000$. Ethiopia's dentist-topopulation ratio has historically been extremely low at 1:1.5 million population. Besides, inter-regional disparities are common and particularly between urban and rural areas (21). The relatively recent establishment of dental education in Ethiopia with three public dental schools across the country and the private education sector in the capital means that numbers are growing, and the country is no longer completely reliant on people studying abroad. However, current workforce data are not available. In addition, as with other sub-Saharan African countries, Ethiopia loses its high-end healthcare professionals due to migration to more developed countries, and the supply does not match the demand because of the low retention $(23,24)$. This, together with the wider social determinants (25), such as individual lifestyle, community influences, working and living conditions; and the more general social conditions in Ethiopia (26), contribute to the volume of untreated disease including oral conditions (3). 
The 'Health System Strengthening in sub-Saharan Africa - ASSET' project in Ethiopia is led by King's College London within the NIHR Global Health Research grant. It is a collaborative project with Addis Ababa University, and it provides an opportunity to support the country's healthcare system through health system strengthening interventions across its three phases: diagnostic, piloting and implementation. The aim of the dental component within the diagnostic phase of the surgical care survey was to examine the burden of oral disease and explore access to dental care and its possible barriers in a rural and urban population within Ethiopia to inform health system strengthening. This will be facilitated using the baseline information to inform the second and third phases of the ASSET project successively.

In light of the above, the aim of this paper is to report the burden and impact of dental caries experience and to investigate access to dental care amongst adults within Ethiopia.

\section{Methods And Materials}

This community oral health survey was part of the surgical care survey of the ASSET project in Ethiopia. Whilst we collected epidemiological data from children and adults using standardised questionnaires and clinical oral examinations during March 2019, this paper includes data from adults only.

\section{Study Area and Population}

The study was conducted in districts across the Butajira Health and Demographic Surveillance Site (HDSS), south-central Ethiopia. The Butajira HDSS is found in Meskan and Mareko districts in the Gurage zone, Southern Nations, Nationalities, and Peoples' Region (SNNPR) of Ethiopia. It covers nine rural and one urban sites, with a total of 40,000 people and an area of approximately $797 \mathrm{~km}^{2}$, of which $9 \mathrm{~km}^{2}$ is Butajira town - the only urban site of the HDSS. Butajira is located $130 \mathrm{~km}$ south of the capital Addis Ababa. The main source of income for the rural population in the HDSS is farming. The production mainly includes Teff (a cereal staple), barley, millet, legumes and maize on the rich soil of the area, with the staple food of Enset (false banana). Butajira also grows coffee, chilli peppers and Khat (a mild stimulant drug) as cash crops (27). This area was chosen for the project as it has an established rural health programme and it provides a study base for essential health research and intervention.

The sample population for this study was drawn from and stratified across the seven accessible sub-districts (kebeles) of Butajira town and Meskan district (woreda) using stratified random sampling of individuals and households. Civil unrest prevented access to the remaining HDSS sites. The sample size, calculated for the ASSET surgical community survey, was 1200: 2 persons of any age, randomly selected from 600 households.

Participants included in this study were those living in Butajira HDSS for at least 1 year prior to the survey and able to communicate in the working language of Amharic. The adult participants were those willing to participate in the study and provide written consent. People with serious health conditions, making it difficult to comply with the interview and oral examination, were excluded from the study.

\section{Instruments and Measures}

The 5th edition of World Health Organisation (WHO) Oral Health Survey Methods (5th ed.) (28) formed the basis for the survey methodology and the standard instruments (electronic questionnaire and data collection forms) were developed drawing on the latest epidemiological research methods: the UK (England, Wales and Northern Ireland) adult dental health survey 2009 (29), and the International Caries Detection and Assessment System (ICDAS) dental caries scoring system (30).

Dental caries experience at tooth level, and the consequences of untreated caries are the main dependent variables. Dental caries experience was determined using the globally accepted DMFT index (the presence of Decayed, Missed and Filled Teeth) extracted from the dentition status data (28). Assessment of dental caries was based on the WHO survey guideline 'tooth condition' assessment format (28), and the threshold of individual lesions was determined using the International Caries Classification and Management System (ICCMS), ICDAS coding criteria. We considered ICDAS 4 ( $\left.\mathrm{D}_{4}\right)$, and above as 'caries', because ICDAS $3\left(D_{3}\right)$ and below is more difficult to assess when only visual examination is used without clinical/diagnostic facilities (30), particularly in areas where Khat chewing is common, as it can stain teeth after repeated use making early caries mode difficult to identify. Khat, a green leaf chewed for its mild stimulating effect, is commonly used in East-Africa and Middle-east region $(31,32)$.

To determine the consequences of untreated caries, we used the PUFA index, presence of Pulpal involvement, Ulcer, Fistula and Abscess (PUFA) (33). We also assessed the presence of pain or any condition related to the chronic complication of dental caries as an additional outcome variable (29).

Sociodemographic factors included in the baseline surgical questionnaire instrument as important independent variables for subsequent analysis were as follows: age, sex, education status, religious affiliation, marital status and location (urban/rural) as these variables are common predictors of caries (34, 35). In relation to lifestyle and behavioural factors, frequency of tooth cleaning and materials used, including toothpaste were collected in the dental data collection instrument and used as proxies for oral hygiene/plaque removal and fluoride exposure $(36,37)$. Other health behavioural and lifestyle factors examined because of their influence on oral health were: Khat chewing, sugar, tobacco and alcohol consumption $(9,38)$. Self-reported dental attendance, which refers to attending a recognised dental service provider, was also obtained to look at access to dental care and possible barriers to care (14, 39).

\section{Standardization}

The dental questionnaire instrument was translated into the working language of the area - Amharic. It was also back translated into English by two native Amharic speakers with full professional competencies in English. They were non-dental/medical professionals with no knowledge about the survey. The original and translated questionnaires were then assessed if they achieve semantic and conceptual equivalence, and it did not require any further amendment. 
The clinical examiners (dentists) were trained over a two-day period and calibrated for identification and assessment of dental caries on the same set of teeth. Inter-examiner agreement was computed using the kappa statistics, with a range of 0.6-1.0 (mean: 0.96). A standard form was created online for recording of the clinical data. Repeat examinations were not possible because of time and logistical constraints.

\section{Data Collection}

Lay data transcribers and dentists teamed up with their surgical community survey counterparts during the data collection. Participants were interviewed faceto-face and examined for oral diseases in their respective households. The transcribers recorded all information (questionnaire and clinical oral examination) using the Open Data Kit (ODK) software on smart phones. Oral examination was visual, conducted using standard disposable dental mirrors and blue-white spectrum solar headtorches with the examinee supine. Dental probes were only used for removing debris, when required.

\section{Data Entry, Cleaning and Analysis}

The ASSET data specialist exported the data and merged the surgical and dental data using unique identifier codes to form the datasets for analysis. We then cleaned the dental data removing any incomplete cases related to this analysis, created new variables and undertook data analysis using SPSS version 25. The numerical variable-age was recoded into six age-bands (28), and the variable dental caries experience was categorized in to $D_{4} M F T=0$ and $D_{4} M F T \geq 1$ prior to the data analysis. Sugar consumption 'risk' was also calculated based on the frequency of reported intake of sweets, cakes, biscuits and soft/fizzy drinks. Consuming at least one of them more than once a week was categorised as 'higher-risk', between once a month and once a week as 'moderate-risk', and rarely or never as 'lower-risk' consumption.

The sociodemographic characteristics, health behaviours and other relevant data were summarized using descriptive statistics. We used frequencies and proportions to describe the variables and used a chi-square test to examine the association between the independent and dependent variables outlined above. The association of health behaviours, tooth cleaning habit and the sociodemographic factors with dental caries experience were further analysed using both unadjusted and adjusted binary logistic regression models.

\section{Results}

Of the 1071 people who participated in the study (89.3\% response rate), 650 were adults. Twenty-four adult participants (3.8\%) were excluded from the final analysis because of missing relevant information. Therefore 626 participants, with an average age of 38.4 years (range: 18-100; sd: 16.4 years) were included for analysis. A higher proportion of the study population was female $(63.9 \%, n=400)$, married $(71.4 \%, n=447)$, and Muslim (76\%, $n=476)$. Just over half $(53.2 \%, n=333)$ lived in rural areas and $44.4 \%(n=278)$ had not received any formal education.

Reported health behaviours were largely positive. The vast majority $(97.9 \%, n=613)$ reported never smoking cigarettes or drinking alcohol $(90.4 \%, \mathrm{n}=566)$, or chewing Khat $(59.0 \%, n=370)$; sugar consumption was generally low with only $10.7 \%(n=67)$ in the 'higher-risk' category (consuming any sweet more than once a week). However, tooth cleaning twice a day or more was only practiced by about one in five adults, as was toothpaste use. Chew-sticks (Mefakiya) were most commonly used for tooth cleaning $(45.0 \%, \mathrm{n}=281)$.

A majority $(74.0 \%, n=463)$ of participants had never been to a dental service. The main reported reason for not attending a dental service was never experiencing any dental pain/problem $(71.3 \%, n=330)$, followed by the distance to a dental clinic/service $(9.3 \%, n=58)$ and the cost of treatment $(8.2 \%, n=$ 51). Only $37.1 \%(n=140)$ of those with dental caries experience reported attending a dental service.

Dental caries experience $\mathrm{D}_{4} \mathrm{MFT}$ and its consequences in relation to demographic and behavioural variables are presented in Table 1. Six out of ten (60.2\%, $\mathrm{n}$ $=337)$ of adults surveyed had a $D_{4}$ MFT score of 1 or more, out of which $88.0 \%(n=332)$ had at least one untreated obvious carious tooth $\left(D_{4} T\right)$ which accounts for $53.0 \%$ of the total study population. 
Table 1

Bivariate analysis of dental caries experience and consequences of untreated dental caries $\left(\mathrm{D}_{4} \mathrm{MFT} \geq 1\right)^{\mathrm{a}}$ with sociodemographic and relevant health behal factors of adults in Butajira HDSS, south-central Ethiopia

\begin{tabular}{|c|c|c|c|c|c|c|c|c|c|c|c|c|c|c|c|c|c|}
\hline \multirow[t]{2}{*}{ Factors } & & \multicolumn{2}{|l|}{ Total } & \multicolumn{3}{|c|}{$\mathrm{DT} \geq 1$} & \multicolumn{3}{|c|}{$\mathrm{MT} \geq 1$} & \multicolumn{3}{|c|}{$\mathrm{DMFT} \geq 1$} & \multicolumn{3}{|c|}{ Pain/Discomfort } & \multicolumn{2}{|c|}{ PUFA $\geq 1$} \\
\hline & & $\mathbf{N}$ & $\%$ & $\mathbf{n}$ & $\%$ & $\begin{array}{l}\mathrm{p}- \\
\text { value }\end{array}$ & $\mathbf{N}$ & $\%$ & $\begin{array}{l}\mathrm{p}- \\
\text { value }\end{array}$ & $\mathbf{N}$ & $\%$ & $\begin{array}{l}\mathrm{p}- \\
\text { value }\end{array}$ & $\mathbf{N}$ & $\%$ & $\begin{array}{l}\mathrm{p}- \\
\text { value }\end{array}$ & $\mathrm{n}$ & $\%$ \\
\hline Total & & 626 & 100 & 332 & 53.0 & & 188 & 30 & & 377 & 60.2 & & 103 & 16.5 & & 45 & 7.2 \\
\hline \multirow[t]{6}{*}{ Age group } & $18-24$ & 123 & 19.7 & 35 & 28.5 & 0.001 & 5 & 4.0 & 0.001 & 36 & 29.3 & 0.001 & 8 & 6.5 & 0.001 & 7 & 5.7 \\
\hline & $25-34$ & 190 & 30.4 & 70 & 36.8 & & 39 & 20.5 & & 85 & 44.7 & & 22 & 11.6 & & 13 & 6.8 \\
\hline & $35-44$ & 103 & 16.5 & 65 & 63.1 & & 30 & 29.1 & & 78 & 75.7 & & 17 & 16.5 & & 3 & 2.9 \\
\hline & $45-54$ & 79 & 12.6 & 56 & 54.4 & & 36 & 35.0 & & 61 & 59.2 & & 18 & 22.8 & & 8 & 10.1 \\
\hline & $55-64$ & 74 & 11.8 & 59 & 79.7 & & 40 & 54.0 & & 64 & 86.5 & & 26 & 35.1 & & 9 & 12.2 \\
\hline & $65+$ & 57 & 9.1 & 47 & 82.5 & & 38 & 66.7 & & 53 & 93.0 & & 12 & 21.1 & & 5 & 8.8 \\
\hline \multirow[t]{2}{*}{ Sex } & Female & 400 & 63.9 & 213 & 53.3 & 0.886 & 126 & 31.5 & 0.165 & 240 & 60.0 & 0.932 & 65 & 16.3 & 0.911 & 28 & 6.0 \\
\hline & Male & 226 & 36.1 & 119 & 52.7 & & 62 & 27.4 & & 137 & 60.6 & & 38 & 16.8 & & 17 & 7.5 \\
\hline \multirow[t]{4}{*}{$\begin{array}{l}\text { Education } \\
\text { level }\end{array}$} & $\begin{array}{l}\text { No formal } \\
\text { education }\end{array}$ & 278 & 44.4 & 205 & 73.7 & 0.001 & 121 & 43.5 & 0.001 & 226 & 81.3 & 0.001 & 65 & 23.0 & 0.001 & 19 & 6.8 \\
\hline & $\begin{array}{l}\text { Primary } \\
\text { school }\end{array}$ & 202 & 32.3 & 88 & 43.6 & & 47 & 23.3 & & 102 & 50.5 & & 23 & 11.4 & & 16 & 7.9 \\
\hline & $\begin{array}{l}\text { Secondary } \\
\text { school }\end{array}$ & 97 & 15.5 & 26 & 26.8 & & 14 & 14.4 & & 34 & 35.1 & & 11 & 11.3 & & 8 & 8.3 \\
\hline & $\begin{array}{l}\text { Higher } \\
\text { education }\end{array}$ & 49 & 7.8 & 13 & 26.5 & & 6 & 12.2 & & 16 & 32.7 & & 4 & 8.16 & & 2 & 4.1 \\
\hline \multirow[t]{3}{*}{ Religion } & Muslim & 476 & 76.0 & 261 & 54.8 & 0.183 & 149 & 31.3 & 0.135 & 296 & 62.2 & 0.166 & 76 & 16.0 & 0.810 & 31 & 6.5 \\
\hline & Orthodox & 131 & 21.0 & 62 & 47.3 & & 37 & 28.2 & & 72 & 55.0 & & 24 & 18.3 & & 12 & 9.2 \\
\hline & Protestant & 19 & 3.0 & 8 & 42.1 & & 2 & 10.5 & & 9 & 47.4 & & 3 & 15.8 & & 2 & 10.5 \\
\hline \multirow[t]{3}{*}{$\begin{array}{l}\text { Marital } \\
\text { Status }\end{array}$} & $\begin{array}{l}\text { Single/ } \\
\text { Never } \\
\text { Married }\end{array}$ & 106 & 16.9 & 30 & 28.3 & 0.001 & 10 & 33.3 & 0.001 & 32 & 30.2 & 0.001 & 8 & 7.6 & 0.017 & 2 & 1.9 \\
\hline & Married & 447 & 71.4 & 242 & 54.1 & & 134 & 55.4 & & 278 & 62.2 & & 79 & 17.7 & & 37 & 8.3 \\
\hline & $\begin{array}{l}\text { Formerly } \\
\text { Married }\end{array}$ & 73 & 11.7 & 60 & 82.2 & & 44 & 60.3 & & 67 & 91.8 & & 16 & 21.9 & & 6 & 8.2 \\
\hline \multirow[t]{2}{*}{ Location } & Urban & 293 & 46.8 & 127 & 43.3 & 0.001 & 81 & 27.7 & 0.222 & 154 & 52.6 & 0.001 & 37 & 19.8 & 0.015 & 23 & 7.9 \\
\hline & Rural & 333 & 53.2 & 205 & 61.6 & & 107 & 32.1 & & 223 & 66.8 & & 66 & 23.0 & & 22 & 6.6 \\
\hline \multirow{2}{*}{$\begin{array}{l}\text { Dental } \\
\text { attendance }\end{array}$} & Never & 463 & 74.0 & 212 & 45.8 & 0.001 & 100 & 21.6 & 0.001 & 237 & 51.2 & 0.001 & 66 & 14.3 & 0.012 & 26 & 5.6 \\
\hline & Ever & 163 & 26.0 & 120 & 73.6 & & 88 & 54.0 & & 140 & 85.9 & & 37 & 22.7 & & 19 & 11.7 \\
\hline \multirow[t]{2}{*}{ Factors } & & \multicolumn{2}{|l|}{ Total } & \multicolumn{3}{|c|}{$\mathrm{DT} \geq 1$} & \multicolumn{3}{|c|}{$\mathrm{MT} \geq 1$} & \multicolumn{3}{|c|}{$\mathrm{DMFT} \geq 1$} & \multicolumn{3}{|c|}{ Pain/Discomfort } & \multicolumn{2}{|c|}{ PUFA $\geq 1$} \\
\hline & & $\mathbf{N}$ & $\%$ & $\mathrm{n}$ & $\%$ & $\begin{array}{l}\mathrm{p}- \\
\text { value }\end{array}$ & $\mathbf{N}$ & $\%$ & $\begin{array}{l}\mathrm{p}- \\
\text { value }\end{array}$ & $\mathbf{N}$ & $\%$ & $\begin{array}{l}\mathrm{p}- \\
\text { value }\end{array}$ & $\mathbf{N}$ & $\%$ & $\begin{array}{l}\mathrm{p}- \\
\text { value }\end{array}$ & $n$ & $\%$ \\
\hline \multirow{4}{*}{$\begin{array}{l}\text { Frequency of } \\
\text { tooth } \\
\text { cleaning }\end{array}$} & $\begin{array}{l}\text { Never/ } \\
\text { Rarely }\end{array}$ & 65 & 10.4 & 48 & 73.9 & 0.001 & 27 & 41.5 & 0.007 & 51 & 78.5 & 0.001 & 9 & 13.9 & 0.001 & 2 & 3.1 \\
\hline & $\begin{array}{l}\text { Less than } \\
\text { once a day }\end{array}$ & 305 & 48.7 & 156 & 51.2 & & 79 & 25.9 & & 178 & 58.4 & & 50 & 16.4 & & 15 & 4.9 \\
\hline & Once a day & 135 & 21.6 & 53 & 39.3 & & 35 & 25.9 & & 65 & 48.2 & & 15 & 11.1 & & 8 & 5.9 \\
\hline & $\begin{array}{l}\text { Twice or } \\
\text { more per } \\
\text { day }\end{array}$ & 121 & 19.3 & 75 & 62.0 & & 47 & 38.8 & & 83 & 68.6 & & 83 & 68.6 & & 20 & 16.5 \\
\hline Tooth & $\begin{array}{l}\text { Mefakiya/ } \\
\text { Chewstick }^{\mathrm{b}}\end{array}$ & 281 & 44.9 & 150 & 53.4 & 0.001 & 87 & 31.0 & 0.004 & 169 & 60.1 & 0.001 & 53 & 18.9 & 0.288 & 20 & 7.1 \\
\hline
\end{tabular}

a DT: ICDAS 4 and above ${ }^{b}$ Mefakiya: an Amharic name for chew-stick

${ }^{\mathrm{c}}$ More than once a week ${ }^{\mathrm{d}}$ Between once a month and once a week ${ }^{\mathrm{e}}$ Rarely or never 


\begin{tabular}{|c|c|c|c|c|c|c|c|c|c|c|c|c|c|c|c|c|c|}
\hline \multirow[t]{2}{*}{ Factors } & & \multicolumn{2}{|c|}{ Total } & \multicolumn{3}{|c|}{$\mathrm{DT} \geq 1$} & \multicolumn{3}{|c|}{$\mathrm{MT} \geq 1$} & \multicolumn{3}{|c|}{ DMFT $\geq 1$} & \multicolumn{3}{|c|}{ Pain/Discomfort } & \multicolumn{2}{|c|}{ PUFA $\geq 1$} \\
\hline & & $\mathrm{N}$ & $\%$ & $\mathrm{n}$ & $\%$ & $\begin{array}{l}\mathrm{p}- \\
\text { value }\end{array}$ & $\mathbf{N}$ & $\%$ & $\begin{array}{l}\mathrm{p}- \\
\text { value }\end{array}$ & $\mathbf{N}$ & $\%$ & $\begin{array}{l}\text { p- } \\
\text { value }\end{array}$ & $\mathbf{N}$ & $\%$ & $\begin{array}{l}\text { p- } \\
\text { value }\end{array}$ & $\mathrm{n}$ & $\%$ \\
\hline & $\begin{array}{l}\text { Wooden } \\
\text { toothpicks }\end{array}$ & 142 & 22.7 & 75 & 52.8 & & 45 & 31.7 & & 89 & 62.7 & & 22 & 15.5 & & 7 & 4.9 \\
\hline & Toothbrush & 114 & 18.2 & 44 & 38.6 & & 19 & 16.7 & & 52 & 45.6 & & 13 & 11.4 & & 10 & 3.1 \\
\hline & Other & 24 & 3.8 & 15 & 62.5 & & 10 & 41.7 & & 16 & 66.7 & & 6 & 25.0 & & 6 & 7.1 \\
\hline & Do not clean & 65 & 10.4 & 48 & 73.9 & & 27 & 41.5 & & 51 & 78.5 & & 9 & 13.8 & & 2 & 4.9 \\
\hline \multirow{3}{*}{$\begin{array}{l}\text { Use of } \\
\text { toothpaste }\end{array}$} & Yes & 118 & 18.9 & 42 & 35.6 & 0.001 & 17 & 14.4 & 0.001 & 50 & 42.4 & 0.001 & 14 & 11.9 & 0.260 & 8 & 8.8 \\
\hline & No & 443 & 70.8 & 242 & 54.6 & & 144 & 32.5 & & 276 & 62.3 & & 80 & 18.1 & & 35 & 25.0 \\
\hline & Do not clean & 65 & 10.4 & 48 & 73.9 & & 27 & 41.5 & & 51 & 78.5 & & 9 & 13.8 & & 2 & 3.1 \\
\hline \multirow{4}{*}{$\begin{array}{l}\text { Frequency of } \\
\text { Khat } \\
\text { Chewing }\end{array}$} & Never Chew & 370 & 59.1 & 164 & 44.3 & 0.001 & 90 & 24.3 & 0.003 & 193 & 52.2 & 0.001 & 54 & 14.6 & 0.484 & 23 & 6.2 \\
\hline & $\begin{array}{l}\text { Less than } \\
\text { weekly }\end{array}$ & 72 & 11.5 & 43 & 59.7 & & 26 & 36.1 & & 48 & 66.7 & & 13 & 18.1 & & 8 & 11.1 \\
\hline & $\begin{array}{l}\text { More than } \\
\text { once a week }\end{array}$ & 101 & 16.1 & 64 & 63.4 & & 40 & 39.6 & & 70 & 69.3 & & 19 & 18.8 & & 8 & 7.9 \\
\hline & $\begin{array}{l}\text { Every day or } \\
\text { nearly every } \\
\text { day }\end{array}$ & 83 & 13.3 & 61 & 73.5 & & 32 & 38.6 & & 66 & 79.5 & & 17 & 20.5 & & 6 & 7.2 \\
\hline \multirow{3}{*}{$\begin{array}{l}\text { Level of } \\
\text { sugar } \\
\text { consumption } \\
\text { risk }\end{array}$} & Higher $^{c}$ & 67 & 10.7 & 31 & 46.3 & 0.004 & 13 & 19.4 & 0.017 & 35 & 52.2 & 0.001 & 9 & 2.0 & 0.733 & 4 & 0.1 \\
\hline & Moderate $^{\mathrm{d}}$ & 223 & 35.6 & 102 & 45.7 & & 59 & 26.5 & & 115 & 51.6 & & 36 & 0.4 & & 20 & 20.0 \\
\hline & Lower $^{\mathrm{e}}$ & 336 & 53.7 & 199 & 59.2 & & 116 & 34.5 & & 227 & 67.6 & & 58 & 68.2 & & 21 & 24.7 \\
\hline \multirow{4}{*}{$\begin{array}{l}\text { Toothache or } \\
\text { discomfort } \\
\text { (in past } 12 \\
\text { months) }\end{array}$} & Often & 44 & 7.0 & 37 & 84.1 & 0.001 & 24 & 54.6 & 0.001 & 38 & 86.4 & 0.001 & 22 & 0.5 & 0.001 & 12 & 27.3 \\
\hline & Occasionally & 133 & 21.3 & 104 & 78.2 & & 58 & 43.6 & & 113 & 85.0 & & 50 & 37.6 & & 14 & 10.5 \\
\hline & Rarely & 97 & 15.5 & 66 & 68.0 & & 35 & 36.1 & & 75 & 77.3 & & 14 & 14.4 & & 4 & 4.1 \\
\hline & Never & 352 & 56.2 & 125 & 35.5 & & 71 & 20.2 & & 151 & 42.9 & & 16 & 4.6 & & 14 & 4.1 \\
\hline
\end{tabular}

a DT: ICDAS 4 and above ${ }^{b}$ Mefakiya: an Amharic name for chew-stick

${ }^{\mathrm{c}}$ More than once a week ${ }^{\mathrm{d}}$ Between once a month and once a week ${ }^{\mathrm{e}}$ Rarely or never

Table 1. Bivariate analysis of dental caries experience and consequences of untreated dental caries $\left(D_{4} M F T \geq 1\right)^{a}$ with sociodemographic and relevant health behavioural factors of adults in Butajira HDSS, south-central Ethiopia

$\mathrm{D}_{4}$ MFT scores ranged from 0-28 (mean, $\left.4.4 \pm 6.9\right)$, and the score increased with age (Fig. 1). Only $0.8 \%(n=3)$ of adults with dental caries experience had fillings, but $49.9 \%(n=188)$ of them had missing teeth. Pain and/or discomfort was reported by $16.5 \%(n=103)$, with $7.2 \%(n=45)$ having one or more PUFA component. Most $(59.9 \%, \mathrm{n}=226)$ adults with dental caries experience reported some tooth pain and/or discomfort during the last year.

\section{Fig. Progression of dental caries experience (DT: ICDAS 4 and above) across the age categories of adults in Butajira HDSS, South-central Ethiopia}

Based on the bivariate analysis, increasing age had a significant and positive association with dental caries experience; whereas educational level was negatively associated. Marital status and location also demonstrated highly significant associations with caries ( $p<0.001$ for all). However, no association was observed with sex and religion. Regarding the health behaviours, dental attendance, frequency of tooth cleaning and materials used (including the use of toothpaste), sugar intake and Khat chewing were highly associated with dental caries experience $(P<0.001$ for all). There were also significant associations between the health impact, notably tooth pain/discomfort related to untreated dental caries and age, education, marital status, dental attendance, location and tooth cleaning frequency $(p<0.05)$. Out of all the independent variables, only dental attendance, tooth cleaning frequency and materials used for dental hygiene were significantly associated with PUFA $(p<0.05)$.

The binary logistic regression models for dental caries experience and predictors are presented in Table 2. In the unadjusted model, all the independent variables, except for sex, had significant associations with dental caries experience $(p<0.05)$. In the adjusted model, the odds of experiencing dental caries were higher in older age-groups, whilst the odds of dental caries experience were lower in higher levels of education. The odds of experiencing dental caries amongst those who reported attending dental services were seven times $(95 \% \mathrm{Cl}: 3.9-12.1, \mathrm{p}=0.001)$ the odds of those who had never attended. Khat was the only lifestyle behavioural component that remained significant in the adjusted model; people who reported chewing Khat daily or nearly every day had four times ( $95 \% \mathrm{Cl}: 1.7-8.3, \mathrm{p}=0.001)$ the odds of experiencing dental caries as compared with reported non-chewers. Location of residence and other health behaviours including tooth cleaning, the risk of sugar intake, did not predict caries experience after adjusting for other factors. 
Table 2

Factors associated with dental caries experience $\left(\mathrm{D}_{4} \mathrm{MFT} \geq 1\right)^{\mathrm{a}}$ among adults in Butajira HDSS, South-central Ethiopia

\begin{tabular}{|c|c|c|c|c|c|}
\hline \multirow[t]{2}{*}{ Explanatory Variables } & & \multicolumn{2}{|c|}{ Unadjusted Model } & \multicolumn{2}{|c|}{ Adjusted Model } \\
\hline & & OR $^{f}$ & $95 \% \mathrm{Cl}$ & $O^{f}$ & $95 \% \mathrm{Cl}$ \\
\hline \multirow[t]{6}{*}{ Age group } & $18-24$ & 1 & [Reference] & 1 & [Reference] \\
\hline & $25-34$ & $1.96^{* *}$ & $1.21-3.17$ & 1.09 & $0.61-1.97$ \\
\hline & $35-44$ & $7.54^{\star \star \star}$ & $4.16-13.67$ & $2.89 * *$ & $1.36-6.14$ \\
\hline & $45-54$ & $8.19 * * *$ & $4.26-15.75$ & $3.24 * *$ & $1.41-7.43$ \\
\hline & $55-64$ & $15.47^{\star \star \star}$ & $7.15-33.45$ & $3.95^{\star \star}$ & $1.54-10.11$ \\
\hline & $65+$ & $32.02^{\star \star \star}$ & $10.79-95.05$ & $10.56^{\star \star \star}$ & $3.14-35.43$ \\
\hline \multirow[t]{2}{*}{ Sex } & Female & 1 & [Reference] & 1 & [Reference] \\
\hline & Male & 1.03 & $0.74-1.43$ & 1.29 & $0.75-2.20$ \\
\hline \multirow[t]{4}{*}{ Educational status } & No formal education & 1 & [Reference] & 1 & [Reference] \\
\hline & Primary school & $0.24^{\star \star \star}$ & $0.16-0.35$ & $0.35^{\star \star \star}$ & $0.20-0.62$ \\
\hline & Secondary school & $0.12^{\star \star \star}$ & $0.07-0.20$ & $0.24^{\star \star \star}$ & $0.11-0.51$ \\
\hline & Higher education & $0.12^{\star \star \star}$ & $0.06-0.22$ & $0.22 * \star \star$ & $0.09-0.55$ \\
\hline \multirow[t]{2}{*}{ Location } & Urban & 1 & [Reference] & 1 & [Reference] \\
\hline & Rural & $1.83^{\star \star \star}$ & $1.32-2.53$ & 0.98 & $0.61-1.58$ \\
\hline \multirow[t]{2}{*}{ Dental attendance } & Never & 1 & [Reference] & 1 & [Reference] \\
\hline & Ever & $5.80^{\star \star \star}$ & $3.60-9.35$ & $6.83^{\star \star \star}$ & $3.86-12.07$ \\
\hline \multirow[t]{4}{*}{ Frequency of tooth cleaning } & Never/ Rarely & 1 & [Reference] & 1 & [Reference] \\
\hline & Less than once a day & $0.39 \star \star$ & $0.20-0.73$ & 0.92 & $0.32-2.60$ \\
\hline & Once a day & $0.26 * \star \star$ & $0.13-0.50$ & 0.78 & $0.27-2.26$ \\
\hline & Twice or more per day & 0.60 & $0.30-1.21$ & 1.48 & $0.50-4.45$ \\
\hline \multirow[t]{5}{*}{ Tooth cleaning material } & Toothbrush & 1 & [Reference] & 1 & [Reference] \\
\hline & Mefakiya/ Chew-stick ${ }^{b}$ & $1.80 \star \star$ & $1.16-2.79$ & 0.62 & $0.20-1.86$ \\
\hline & Wooden toothpicks & 2.00 ** & $1.21-3.31$ & 0.52 & $0.16-1.74$ \\
\hline & Other & 2.39 & $0.95-6.02$ & 0.75 & $0.16-3.44$ \\
\hline & Don't clean & $4.34^{\star \star \star}$ & $2.16-8.72$ & 1.20 & $0.36-3.98$ \\
\hline \multirow[t]{2}{*}{ Use of toothpaste } & Yes & 1 & [Reference] & 1 & [Reference] \\
\hline & No & $2.25^{\star \star \star}$ & $1.49-3.42$ & 1.33 & $0.446-4.00$ \\
\hline \multirow[t]{4}{*}{ Frequency of Khat chewing } & Never & 1 & [Reference] & 1 & [Reference] \\
\hline & Less than weekly & 1.83 & $1.08-3.12$ & 0.67 & $0.34-1.32$ \\
\hline & More than once a week & $2.07^{\star \star}$ & $1.30-3.31$ & 1.68 & $0.90-3.13$ \\
\hline & Every day/nearly every day & $3.56^{\star * *}$ & $2.01-6.30$ & $3.79 * \star \star$ & $1.73-8.31$ \\
\hline \multirow[t]{3}{*}{ Level of sugar consumption risk } & Higher $^{c}$ & 1 & [Reference] & 1 & [Reference] \\
\hline & Moderate $^{d}$ & 0.97 & $0.56-1.68$ & 0.78 & $0.39-1.56$ \\
\hline & Lower ${ }^{\mathrm{e}}$ & $1.90 *$ & $1.12-3.24$ & 1.30 & $0.63-2.65$ \\
\hline \multicolumn{6}{|c|}{ aDT: ICDAS 4 and above ${ }^{\text {b} M e f a k i y a: ~ a n ~ A m h a r i c ~ n a m e ~ f o r ~ c h e w-s t i c k ~}$} \\
\hline \multicolumn{6}{|c|}{${ }^{c}$ More than once a week ${ }^{d}$ Between once a month and once a week ${ }^{e}$ Rarely or never } \\
\hline \multicolumn{6}{|c|}{ fBinary Logistic Regression was fitted, and Odds Ratio was reported } \\
\hline${ }^{*} p<0.05 ; * \star p<0.01 ; * \star \star p<0.001$ & & & & & \\
\hline
\end{tabular}




\section{Discussion}

In this cross-sectional study amongst a rural and urban population of south-central Ethiopia, six out of ten adults were found to have dental caries experience. This study is one of the few oral health research projects to have been carried out in the country and the first population-based oral health survey to be part of a large multi-disciplinary global health project providing an overview of adult oral health in the community and the most extensive in nature. This research has certain limitations which need to be acknowledged. First, it was conducted in a relatively ethnically homogenous population, which therefore cannot be considered nationally representative. Second, almost half (46.8\%) of the study participants were from the urban area due to the accessibility challenge in some rural areas. Since a large proportion (80.0\%) of the general population in the country is rural, this again needs to be recognised as unrepresentative of the national situation; however, it allowed comparison between the rural and urban settings and given the study findings whereby the differences between urban and rural settings were not significantly different in the adjusted logistic regression model, this may not be a major factor. Third, the people who stayed at home and were available to participate in the data collection might be different from those who were out engaging in work and other activities and thus possibly overrepresented. However, every effort was made to ensure that people who worked away from home were included in the study, although most men working in the fields might have been missed. Fourth, the questionnaire data were collected by face-to-face interview, which means participants may have given more socially desirable answers about their lifestyle and behaviours. None-the-less, given the very low level of education in this community, this was the most appropriate survey method. Fifth, the sample size was calculated based on surgical care needs; however as surgical care needs are less common than oral diseases, this provided a substantial sample for the oral health survey when compared with other national dental surveys (29), and our findings will provide a sound basis to inform future epidemiological studies in Ethiopia.

The magnitude of untreated obvious caries (clinically visible lesions representing demineralisation in the middle third of the dentine, and above indicated by the ICDAS 4 threshold) in the study participants was high; if the early and sub-clinical lesions were included, which would require optimal clinical and radiographic examinations (40), a higher value would result. These findings suggest an increasing burden of NCDs in general (41). Interestingly, a higher proportion of dental caries experience was observed in rural areas, unlike a previous study in Ethiopia which reported a higher prevalence of caries in urban areas (61.1\%) (14). However, the odds of experiencing dental caries were almost the same for both locations in the adjusted logistic regression model. Although rural areas are generally suggested to have less access to cariogenic foods, this may be changing with the nutrition transition in Africa; in addition to the limited access to dental care and preventive services as compared to urban areas. Similar issues exist in both lower and higher income countries, whereby the rural population has more untreated caries $(35,42)$.

In the study participants, the odds of experiencing caries increased with age and the pattern of the condition is also cumulative throughout life by its nature (34). This finding mirrors the emerging result of the Dunedin longitudinal study (the 1972/3 birth cohort) of dental caries trajectory assessment in which the participants dental caries experience in the different categories of the trajectory increased with age (43). However, there was no significant difference between males and females in our study regarding their dental caries experience unlike previous studies in the UK (44), but it is similar with the recent global burden of oral health study findings $(6,45)$.

In this regression model, the odds of experiencing dental caries significantly reduced with increasing educational attainment of adults. Education, being one of the social determinants of health can also influence an individual's position in the society and determine their income. This in turn influences the risk of one's exposure to different diseases (25), including dental caries in adults (34).

Health behaviours, whilst important risk factors for oral disease (9), are less likely to be significant in our model. Amongst the health behaviours assessed in this survey, chewing Khat daily was associated with increased odds of dental caries experience when keeping other variables in the model constant. The association of the oral hygiene practice and sugar consumption was no longer present after the statistical adjustment. However, people who reported cleaning their teeth twice or more per day were those with a higher dental caries experience. This might be explained by the fact that those who already have problem tend to perceive severity of the disease and clean their teeth more (46), but do not appear to be using fluoride toothpaste (47). Khat chewing has been suggested to be associated with some oral conditions including periodontal diseases, attrition of the tooth surface and tooth discolouration, oral cancer and other mucosal diseases. According to Al-Maweri (2018), Khat itself is not cariogenic. However, it has a dehydrating effect on the oral mucosa and a bitter taste. Consequently, Khat chewers tend to consume a large quantity of non-alcoholic fluids, such as carbonated soft drinks, water, and coffee (usually sweetened). Some also supplement the leaves with refined sugar and it also increases the tendency to tobacco smoking (38). A cohort study of 98 Yemeni Khat chewers and 101 non-chewers, aged 18-35 years with early occlusal caries suggested a higher risk of dental caries progression in low-income Khat chewers as compared to the non-chewers, although the study follow-up was short (48).

Whilst dental attendance was generally uncommon, it was notable in those with dental caries experience in our study, only just over one-third had attended a dental service. However, those who had been to the service had seven times the odds of dental caries experience than those who never did. This might suggest that dental visit was perceived need driven and given the level of untreated diseases, care was probably limited to the offending lesion. In this regard, the main reasons for not attending dental services are generally multifactorial (39), and it can also be highlighted by the barriers of access related to the direct and indirect costs. A study of Jordanian adults on factors influencing access to dental care also reported the cost of treatment and perceived lack of treatment need, following lack of time as main reasons for not attending a dental care (49). However, our study participants did not highlight lack of time as a reason, but mainly distance to the dental service and cost of treatments. Another study among adult city-dwellers in Burkina Faso also highlighted cost of dental treatment as the main barrier to dental visits (50). Dental treatment is known to expose households to unpredictable and significant financial costs; recent research has shown, in low income households the larger portion of their disposable income could be used for dental treatment mainly in out-of-pocket payment situations (51). This could also be the case in Ethiopia, as out-of-pocket payment is the main method of funding healthcare (52).

According to our findings, half of the people with dental caries experience had missing teeth due to caries, and less than one percent had dental fillings. This indicates that tooth extraction is the most widely used treatment modality for dental caries in the area. This can be explained by late presentation with 
disease, due to challenges in access to care resulting in limited choice of treatment. However, socioeconomic conditions can also determine the access to preventive and restorative dental treatments (53), in addition to the inequity of healthcare services which is a challenge in most developing countries. However, the use of primary care services helped most industrialized countries to achieve greater equity so that people with greater health needs can get better access to services (54), albeit with less regularity. Reviews of oral health in the African region also suggested those challenges can be addressed by creating universal and equitable access to quality, affordable and appropriate oral health services (55).

This research provides important insights to dental caries experience in Ethiopia and provides pilot data to inform a nationally representative survey of oral health. This will help to inform appropriate workforce capacity building, through modelling future scenarios for dental service provision. It will be important to develop research informed feasible ways of addressing their oral healthcare needs of urban and rural populations to inform health policy on health promotion and healthcare services. Further research should also investigate oral health in relation to NCDs, together with the possible relationship of Khat and dental caries.

\section{Conclusion}

The finding suggests that there is a high burden of dental caries amongst adults in the district of Butajira and marked social inequity. Caries experience increases with age and is less prevalent amongst educated and urban populations. Generally, dental attendance is low and there is a high need for dental care. Overall, this implies a clear need of health system strengthening interventions including oral health promotion.

\section{Declarations}

\section{Funding and Disclaimer}

This research was funded by the National Institute for Health Research (NIHR) Global Health Research Unit on Health System Strengthening in Sub-Saharan Africa, King's College London (GHRU 16/136/54) using UK aid from the UK Government to support global health research. The views expressed in this publication are those of the author(s) and not necessarily those of the NIHR or the Department of Health and Social Care or Public Health England.

JEG, CH and MJP at King's also receive the ASSET project funding. CH additionally receives support from AMARI (African Mental Health Research Initiative) as part of the DELTAS (Developing Excellence in Leadership, Training and Science) Africa Initiative [DEL-15-01]. JEG additionally receives funding from Public Health England (Dental Public Health Academic Funding).

\section{Acknowledgements}

We would like to acknowledge the UK Foreign and Commonwealth Office, Chevening Secretariat for fully funding the lead author's (BB) MSc in Dental Public Health; also, St Paul's Hospital Millennium Medical College, Addis Ababa, Ethiopia and King's College London, London, UK.

\section{Ethical Considerations}

Ethical clearance was obtained from King's College London (HR-17/18-6144) and Addis Ababa University (026/18/PSY). Informed consent was obtained from participants.

\section{Availability of data and materials}

The datasets used and/or analysed during the current study are available from the corresponding author on reasonable request.

\section{Competing Interests}

The authors declare that they have no competing interests.

\section{Consent for publication}

Not applicable

\section{Authors' contributions}

MJP conceived and designed the study, and led the ASSET project bid (CH and JEG were co-applicants); $\mathrm{CH}$ led the ASSET-Ethiopia project and surgical care survey protocol; JEG led the oral/dental health survey; BB and CH contributed to the oral/dental health survey design, and MJP approved it; FE contributed in 
the planning and conducting the study; $\mathrm{CH}$ overlooked the fieldwork locally, and BB and JEG remotely; BB cleaned and analysed the data, and drafted the paper; JEG, $\mathrm{CH}$ and MJP refined the paper. All authors read and have approved the manuscript for submission.

\section{References}

1. Glick M, Williams DM, Kleinman DV, Vujicic M, Watt RG, Weyant RJ. A new definition for oral health developed by the FDI World Dental Federation opens the door to a universal definition of oral health. J Am Dent Assoc. 2016;147(12):915-7.

2. Peres MA, Macpherson LMD, Weyant RJ, Daly B, Venturelli R, Mathur MR, et al. Oral diseases: a global public health challenge. Lancet. 2019;394(10194):249-60.

3. Petersen PE, Kwan S. Equity, social determinants and public health programmes-the case of oral health. Community Dent Oral Epidemiol. 2011;39(6):481-7.

4. Marcenes W, Kassebaum NJ, Bernabe E, Flaxman A, Naghavi M, Lopez A, et al. Global burden of oral conditions in 1990-2010: a systematic analysis. J Dent Res. 2013;92(7):592-7.

5. Jin LJ, Lamster IB, Greenspan JS, Pitts NB, Scully C, Warnakulasuriya S. Global burden of oral diseases: emerging concepts, management and interplay with systemic health. Oral Dis. 2016;22(7):609-19.

6. Kassebaum NJ, Smith AGC, Bernabe E, Fleming TD, Reynolds AE, Vos T, et al. Global, Regional, and National Prevalence, Incidence, and DisabilityAdjusted Life Years for Oral Conditions for 195 Countries, 1990-2015: A Systematic Analysis for the Global Burden of Diseases, Injuries, and Risk Factors. J Dent Res. 2017;96(4):380-7.

7. Gouda HN, Charlson F, Sorsdahl K, Ahmadzada S, Ferrari AJ, Erskine H, et al. Burden of non-communicable diseases in sub-Saharan Africa, 1990-2017: results from the Global Burden of Disease Study 2017. Lancet Glob Health. 2019;7(10):e1375-e87.

8. Kassebaum NJ, Bernabe E, Dahiya M, Bhandari B, Murray CJ, Marcenes W. Global burden of severe periodontitis in 1990-2010: a systematic review and meta-regression. J Dent Res. 2014;93(11):1045-53.

9. Abid A, Maatouk F, Berrezouga L, Azodo C, Uti O, El-Shamy H, et al. Prevalence and Severity of Oral Diseases in the Africa and Middle East Region. Adv Dent Res. 2015;27(1):10-7.

10. International Monetary Fund. World Economic Outlook. Washington, DC, U.S.A.: International Monetary Fund Publication Services; 2019.

11. Central Intelligence Agency. Ethiopia. The World Factbook [Internet]. 2020 17March 2020. Available from: https://www.cia.gov/library/publications/theworld-factbook/geos/et.html.

12. Mulu W, Demilie T, Yimer M, Meshesha K, Abera B. Dental caries and associated factors among primary school children in Bahir Dar city: a cross-sectional study. BMC Res Notes. 2014;7:949.

13. Ayele FA, Taye BW, Ayele TA, Gelaye KA. Predictors of dental caries among children 7-14 years old in Northwest Ethiopia: a community based crosssectional study. BMC Oral Health. 2013;13:7.

14. Tafere $\mathrm{Y}$, Chanie S, Dessie T, Gedamu H. Assessment of prevalence of dental caries and the associated factors among patients attending dental clinic in Debre Tabor general hospital: a hospital-based cross-sectional study. BMC Oral Health. 2018;18(1):119.

15. Burnett D, Aronson J, Asgary R. Oral health status, knowledge, attitudes and behaviours among marginalized children in Addis Ababa, Ethiopia. J Child Health Care. 2016;20(2):252-61.

16. United Nations. Sustainable Development Goals Ethiopia: United Nations Development Programme; 2020 [Available from: https://ethiopia.un.org/en/sdgs.

17. Guideline for implementation of a patient referral system [Internet]. Medical Services Directorate. 2010 [cited 15 November 2018]. Available from: https://www.medbox.org/ethiopia-guideline-for... of... referral-system/download.pdf.

18. Bradley E, Hartwig KA, Rowe LA, Cherlin EJ, Pashman J, Wong R, et al. Hospital quality improvement in Ethiopia: a partnership-mentoring model. Int $J$ Qual Health Care. 2008;20(6):392-9.

19. Prasad M, Manjunath C, Murthy AK, Sampath A, Jaiswal S, Mohapatra A. Integration of oral health into primary health care: A systematic review. J Family Med Prim Care. 2019;8(6):1838-45.

20. Petersen PE. Strengthening of oral health systems: oral health through primary health care. Med Princ Pract. 2014;23(Suppl 1):3-9.

21. Gallagher JE, Hutchinson L. Analysis of human resources for oral health globally: inequitable distribution. Int Dent J. 2018;68(3):183-9.

22. World Health Organization. Oral Health Manpower: Ethiopia Geneva: WHO Prevention of Noncommunicable Diseases (PND); 2019 [Available from: https://www.mah.se/CAPP/Country-Oral-Health-Profiles/AFRO/Ethiopia-/Oral-Health-Manpower/.

23. Gile PP, Buljac-Samardzic M, Klundert JV. The effect of human resource management on performance in hospitals in sub-Saharan Africa: a systematic literature review. Hum Resour Health. 2018;16(1):34.

24. Hagopian A, Thompson MJ, Fordyce M, Johnson KE, Hart LG. The migration of physicians from sub-Saharan Africa to the United States of America: measures of the African brain drain. Hum Resour Health. 2004;2:17.

25. Dahlgren G, Whitehead M. Policies and Strategies to Promote Social Equity in Health Stockholm, Sweden: Institute for Futures Studies; 1991 [Available from: core.ac.uk/download/pdf/6472456.pdf.

26. Baru A, Murugan P. Social determinants of vulnerability to ill-health: Evidences from Mendi Town, Western Ethiopia. J Health Soc Sci. 2016;1(3):263-78.

27. Berhane Y, Wall S, Kebede D, Emmelin A, Enqueselassie F, Byass P, et al. Establishing an epidemiologic al field laboratory in rural areas - potentials for public health research and interventions The Butajira Rural Health Programme 1987-1999. The Ethiopian Journal of Health Development. $1999 ; 13$. 
28. World Health Organization. Oral Health Surveys: Basic Methods. 5th ed. France: World Health Organization; 2013.

29. O' Sullivan I, Lader D, Beavan-Seymour C, Chenery V, Fuller E, Sadler K. Foundation Report: Adult Dental Health Survey 2009 (Technical information). 2011 March 24.

30. Criteria Manual - International Caries Detection and Assessment System (ICDAS II). Revised in December and July 2009 (Bogota, Colombia and Budapest, Hungary) - from a Workshop held in Baltimore, Maryland, March 12th-14th, 2005 [Internet]. ICDAS Foundation. 2009. Available from: http://www.icdas.org.

31. Abebe W. Khat and synthetic cathinones: Emerging drugs of abuse with dental implications. Oral Surg Oral Med Oral Pathol Oral Radiol. 2018;125(2):140-6.

32. Gebissa E. Khat in the Horn of Africa: historical perspectives and current trends. J Ethnopharmacol. 2010;132(3):607-14.

33. Monse B, Heinrich-Weltzien R, Benzian H, Holmgren C, van Palenstein Helderman W. PUFA-an index of clinical consequences of untreated dental caries. Community Dent Oral Epidemiol. 2010;38(1):77-82.

34. Costa SM, Martins CC, Bonfim Mde L, Zina LG, Paiva SM, Pordeus IA, et al. A systematic review of socioeconomic indicators and dental caries in adults. Int J Environ Res Public Health. 2012;9(10):3540-74.

35. Ogunbodede EO, Kida IA, Madjapa HS, Amedari M, Ehizele A, Mutave R, et al. Oral Health Inequalities between Rural and Urban Populations of the African and Middle East Region. Adv Dent Res. 2015;27(1):18-25.

36. Griffin SO, Regnier E, Griffin PM, Huntley V. Effectiveness of fluoride in preventing caries in adults. J Dent Res. 2007;86(5):410-5.

37. Chidzonga MM, Carneiro LC, Kalyanyama BM, Kwamin F, Oginni FO. Determinants of Oral Diseases in the African and Middle East Region. Adv Dent Res. 2015;27(1):26-31.

38. Al-Maweri SA, Warnakulasuriya S, Samran A. Khat (Catha edulis) and its oral health effects: An updated review. J Investig Clin Dent. 2018;9(1).

39. Harris RV, Pennington A, Whitehead M. Preventive dental visiting: a critical interpretive synthesis of theory explaining how inequalities arise. Community Dent Oral Epidemiol. 2017;45(2):120-34.

40. Ismail Al, Pitts NB, Tellez M, Authors of International Caries C, Management S, Banerjee A, et al. The International Caries Classification and Management System (ICCMS) An Example of a Caries Management Pathway. BMC Oral Health. 2015;15(Suppl 1):9.

41. Melaku YA, Temesgen AM, Deribew A, Tessema GA, Deribe K, Sahle BW, et al. The impact of dietary risk factors on the burden of non-communicable diseases in Ethiopia: findings from the Global Burden of Disease study 2013. Int J Behav Nutr Phys Act. 2016;13(1):122.

42. Fos P, Hutchison L. The State of Rural Oral Health: A Literature Review -. Rural Healthy people 2010: A companion document to Healthy People 2010. College Station, TX: The Texas. A \& M University System Health Science Center, School of Rural Public Health, Southwest Rural Health Research Center2003.

43. Broadbent JM, Thomson WM, Poulton R. Trajectory patterns of dental caries experience in the permanent dentition to the fourth decade of life. J Dent Res. 2008;87(1):69-72.

44. Steele JG, Treasure ET, O'Sullivan I, Morris J, Murray JJ. Adult Dental Health Survey 2009: transformations in British oral health 1968-2009. Br Dent J. 2012;213(10):523-7.

45. Kassebaum NJ, Bernabe E, Dahiya M, Bhandari B, Murray CJ, Marcenes W. Global burden of untreated caries: a systematic review and metaregression. J Dent Res. 2015;94(5):650-8.

46. Kasmaei P, Amin Shokravi F, Hidarnia A, Hajizadeh E, Atrkar-Roushan Z, Karimzadeh Shirazi K, et al. Brushing behavior among young adolescents: does perceived severity matter. BMC Public Health. 2014;14:8.

47. Public Health England. Delivering better oral health: an evidence-based toolkit for prevention. In: Health, editor. Third ed. London2017.

48. Al-Alimi KR, Razak AAA, Saub R. Is Khat chewing habit a risk factor for occlusal caries progression? Afr Health Sci. 2018;18(4):1036-45.

49. Obeidat SR, Alsa'di AG, Taani DS. Factors influencing dental care access in Jordanian adults. BMC Oral Health. 2014;14:127.

50. Varenne B, Petersen PE, Fournet F, Msellati P, Gary J, Ouattara S, et al. Illness-related behaviour and utilization of oral health services among adult citydwellers in Burkina Faso: evidence from a household survey. BMC Health Serv Res. 2006;6:164.

51. Bernabe E, Masood M, Vujicic M. The impact of out-of-pocket payments for dental care on household finances in low and middle income countries. BMC Public Health. 2017;17(1):109.

52. Tolla MT, Norheim OF, Verguet S, Bekele A, Amenu K, Abdisa SG, et al. Out-of-pocket expenditures for prevention and treatment of cardiovascular disease in general and specialised cardiac hospitals in Addis Ababa, Ethiopia: a cross-sectional cohort study. BMJ Glob Health. 2017;2(2):e000280.

53. Cheema J, Sabbah W. Inequalities in preventive and restorative dental services in England, Wales and Northern Ireland. Br Dent J. 2016;221(5):235-9.

54. Starfield B. The hidden inequity in health care. Int J Equity Health. 2011;10:15.

55. World Health Organization. Promoting Oral Health in Africa: Prevention and control of oral diseases and noma as part of essential noncommunicable disease interventions. In: Africa WROf, editor. Brazzaville, Republic of Congo 2016.

\section{Figures}




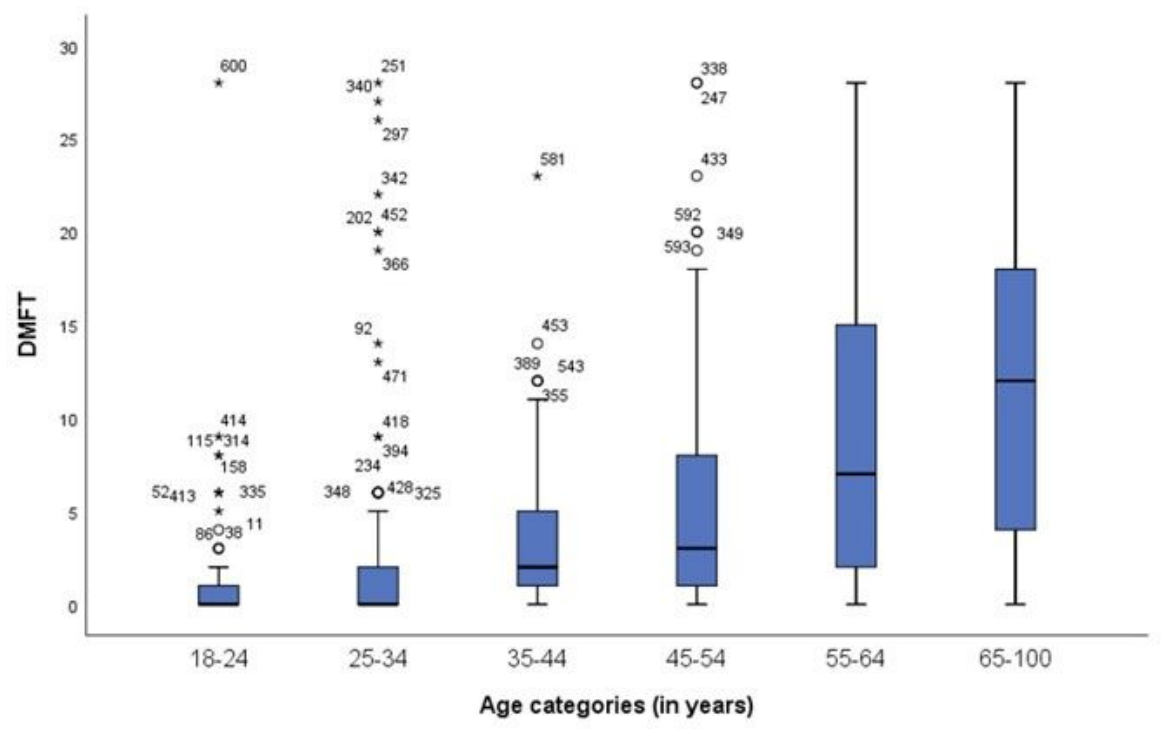

Figure 1

Progression of dental caries experience (DT: ICDAS 4 and above) across the age categories of adults in Butajira HDSS, South-central Ethiopia 